\title{
Electroacupuncture for chemotherapy-induced anorexia through humoral appetite regulation: A preliminary experimental study
}

\author{
KI SUNG KANG ${ }^{1}$, WONSANG HUH ${ }^{1}$, YEOJIN BANG ${ }^{2}$, HYUN JIN CHOI ${ }^{2}$, \\ JI YUN BAEK ${ }^{1}$, JI HOON SONG ${ }^{3}$, JUNG WON KANG ${ }^{4}$ and TAE-HUN KIM ${ }^{5}$ \\ ${ }^{1}$ Department of Preventive Medicine, College of Korean Medicine, Gachon University, Seongnam, \\ Gyeonggi-do 13120; ${ }^{2}$ College of Pharmacy and Institute of Pharmaceutical Sciences, CHA University, \\ Seongnam, Gyeonggi-do 13488; ${ }^{3}$ Department of Medicine, University of Ulsan College of Medicine, \\ Seoul 05505; ${ }^{4}$ Department of Acupuncture and Moxibustion, College of Korean Medicine, Kyung Hee University; \\ ${ }^{5}$ Korean Medicine Clinical Trial Center, Korean Medicine Hospital, Kyung Hee University, Seoul 02447, Republic of Korea
}

Received October 27, 2017; Accepted September 13, 2018

DOI: $10.3892 /$ etm.2019.7250

\begin{abstract}
Chemotherapy-induced anorexia (CIA), which may lead to severe nutrition-associated problems, is a common complication associated with anti-cancer therapies. In the present study, the anti-anorexigenic effect of electroacupuncture (EA) was explored through assessing a change in appetite-associated peptides and c-Fos expression in a rat model of cisplatin-induced anorexia. In order to identify the most effective acupuncture point, 20 male Wistar rats (divided into five groups including the normal saline control, cisplatin only control and three groups according to the acupoints stimulated) were subjected to EA for $10 \mathrm{~min}$ at CV12, ST36 or PC6 daily for 4 days. Subsequently, the rats received intraperitoneal injections of cisplatin $(6 \mathrm{mg} / \mathrm{kg})$ to induce CIA. Food intake and reduction in body weight gain as the anorexia-associated outcomes were assessed daily for up to 3 days after cisplatin injection, and CV12 was eventually chosen as the most effective acupoint to test the anti-anorexigenic effect of EA. Furthermore, food intake, body weight and the concentrations of appetite-associated peptides, including ghrelin, cholecystokinin (CCK) and 5-hydroxytryptamine (5-HT), in addition to c-Fos expression, were comparatively assessed between the CV12 EA group ( $n=6$; rats treated with EA at CV12 daily for 4 days) and a control group ( $n=6$; rats without treatment). The results indicated that the CV12 EA group exhibited a better outcome regarding food intake and body weight compared with the controls. Although there was no statistically significant difference observed, the secretion of serum ghrelin and
\end{abstract}

Correspondence to: Professor Tae-Hun Kim, Korean Medicine Clinical Trial Center, Korean Medicine Hospital, Kyung Hee University, 23 Kyungheedae-ro, Seoul 02447, Republic of Korea E-mail: rockandmineral@gmail.com

Key words: electroacupuncture, chemotherapy-induced anorexia, cisplatin, ghrelin, 5-hydroxytryptamine, cancer, appetite
CCK was increased in the CV12 EA group compared with that in the control group. The plasma level of 5-HT after cisplatin injection in the CV12 EA group was lower compared with that in the control, although no statistical significance was reached. Although not statistically significant, the expression of c-Fos protein in the nucleus tractus solitarius (NTS) was reduced in the CV12 EA rats. In addition, the hypothalamic mRNA levels of brain-derived neurotrophic factor (BDNF) were significantly increased in the CV12 EA group. In the hypothalamus, the expression of neuropeptide Y mRNA slightly increased in the cisplatin + CV12 EA group compared with the cisplatin only control group. In conclusion, the anti-anorexigenic effect of EA on CIA may be associated with an increase in the secretion of ghrelin and CCK and a decrease in the secretion of 5-HT into the serum, a reduction of c-Fos expression in the NTS and an increase in BDNF mRNA expression in the hypothalamus.

\section{Introduction}

Malnutrition due to anorexia is one of the most common problems associated with cancer and anti-cancer therapies. Of all hospitalized cancer patients in Korea, $>50 \%$ have nutritional problems (1), and the worldwide prevalence of nutrition-associated problems in cancer patients displays similar patterns $(2,3)$. For instance, cachexia, a serious malnutrition condition featuring significant irreversible weight loss in patients with serious chronic health conditions, is associated with a poor prognosis, particularly in terms of functional performance in daily life and anti-cancer therapy tolerance (4). Therefore, nutritional support and a proactive treatment strategies for malnutrition itself and associated symptoms are necessary in cancer care to improve clinical outcomes for cancer patients (5).

Cancer-associated nutritional problems may have various origins, and chemotherapy is one of the potential causes of this condition, which is due to the negative effects on gastrointestinal (GI) motility and appetite (4). Chemotherapy-induced nausea and vomiting (CINV) as well as anorexia, which are important 
acute complications during anti-cancer chemotherapy, involve various neurotransmitters and metabolic peptides of the central nervous system (CNS) and GI tract. Serotonin receptor antagonists and steroid medications, which are the most frequently prescribed types of drug for this condition, have been proven to be effective in decreasing acute symptoms, but due to their high cost and limited capacity to prevent late CINV, the use of these drugs is still under debate. Hence, it is essential to identify and develop novel and diverse pharmacological agents and non-drug interventions for CINV and anorexia, which are to be used in future clinical practice (6).

Acupuncture is a non-drug intervention that has been used for managing various conditions in cancer patients. For instance, it is used to reduce hot flush in breast cancer patients (7), relieve various types of cancer-associated pain, including post-operative and malignancy-associated pain (8), as well as to improve cancer-associated fatigue (9) and leukopenia (10). Regarding nutrition-associated problems, clinical research has indicated that acupuncture may reduce the frequency and severity of CINV (11). In a rat model, acupuncture significantly reduced kaolin uptake, which is the most commonly used alternative measure for evaluating nausea and vomiting. Furthermore, acupuncture attenuated the decrease in food intake and body weight due to cisplatin injection through inhibition of duodenal serotonin secretion (12). Although the validity of studies using acupuncture are under debate due to issues including the suitability of placebo (sham) needles as a control intervention and small effect sizes that do not simply exceed non-specific (placebo) effects (13), the clinical effectiveness of acupuncture for anorexia in cancer patients has been convincingly demonstrated $(14,15)$. However, the mechanisms underlying the effects of acupuncture on chemotherapy-induced anorexia have remained to be fully elucidated in an in vivo study.

Ghrelin and cholecystokinin (CCK) are two representative GI hormones, which regulate feeding and may serve as therapeutic targets for anorexia (16). The present study focused on the changes of these two hormones that are mainly associated with feeding regulation as opposed to other hormones, e.g. insulin and leptin, which are involved in metabolic disorders. In the present study, the anti-anorexigenic effects of acupuncture treatment and changes in peptide hormone levels associated with anorexia were assessed as a means of investigating the underlying mechanisms of the efficacy of acupuncture in a rat model of cisplatin-induced anorexia. Electroacupuncture (EA) is a specific type of acupuncture, which stimulates acupuncture points with electric current and is commonly used due to its ease of operation and constant stimulation delivery. We adopted EA as the main intervention for this study.

\section{Materials and methods}

Animals. In total, 32 male Wistar rats (age, 7 weeks; weight, 180-200 g) were obtained from Orient Bio Co., Ltd. (Seongnam, Korea) and used for evaluating the beneficial effect of acupuncture on chemotherapy-induced anorexia (CIA). Rats were housed at $23 \pm 2{ }^{\circ} \mathrm{C}$ and $55 \pm 5 \%$ humidity with a standard 12-h light/dark cycle, and were given free access to water and a normal diet containing $10 \%$ fat for a period of one week after arrival.
Study procedure. The present study comprised two experiments: In Experiment 1, the point-specific effect of electroacupuncture (EA) was assessed to determine the most effective among the potential acupuncture points, including CV12, PC6 and ST36. In Experiment 2, changes in the levels of appetite-associated peptides in the serum and duodenal tissue were evaluated, and changes in c-Fos expression in the brain were detected, in order to define a possible mechanism of the effects of acupuncture. For Experiment 1, 20 rats were randomly allocated into the following five groups according to the acupuncture points/treatments: Normal saline control group with acupuncture stimulation $(n=4)$, cisplatin only control group without acupuncture stimulation (n=4), CV12 EA group $(n=4)$, PC6 EA group $(n=4)$ and ST36 EA group $(n=4)$. The rats were stimulated daily with EA four times in total, namely three times prior and once after cisplatin administration. Rats were housed separately in metabolic cages during the experimental period, and their body weight was assessed daily. The changes of food and water intake, as well as the amount of excreted urine, were also assessed on a daily basis. According to the results from Experiment 1, EA at CV12 was more effective compared with the other points in terms of its effect on food intake and body weight. Thus, CV12 was selected for Experiment 2, which included two experimental groups, cisplatin + CV12 EA $(n=6)$ and cisplatin only control which received EA at a non-acupoint, $(n=6)$. Rats were sacrificed on day 3 after cisplatin administration. The serum and tissue samples were collected and immediately frozen and stored at $-80^{\circ} \mathrm{C}$ until use.

Induction of anorexia. After adaptation, rats were randomly allocated into different experimental groups. Animals were injected intraperitoneally with $6 \mathrm{mg} / \mathrm{kg}$ of cisplatin dissolved in saline at $10 \mathrm{AM}$ on day 0 , as previously described (12).

Measurement of body weight and food intake. For Experiment 1, acrylic metabolic cages (cat. no. JD-C-66, Jeung Do Bio \& Plant, Seoul, Korea) were used to minimize animal stress during the daily assessment of individual body weight, food intake, water intake and urine excretion $(17,18)$. The body weight and food intake of individual rats was measured daily during the 7-day experimental period. During each 24-h period, food intake was measured by collecting, drying and weighing the remaining food in the food container and any spilt in the cage. Body weight, food intake, water intake and urine excretion measurements and acupuncture stimulation were performed every day at $\sim 10 \mathrm{am}$.

EA stimulation. Three acupuncture points, CV12, PC6 and ST36, were selected based on a previous study and expert opinion (12). All acupuncture points were located using the acupuncture map for rats based on a commonly followed text book on acupuncture experiments (19). In the CV12 group, two points (a center of the mid-line and a point $1 \mathrm{~cm}$ below) were selected. In the PC6 group, the two points were 3 and $5 \mathrm{~mm}$ away from the wrist between the radius and ulnar bones in the left anterior forearm. In the ST36 group, the two points were 0.7 and $1.4 \mathrm{~mm}$ away from the base of the patella along the tibia in the left anterior lower leg. Stainless disposable acupuncture needles $(0.25 \times 40 \mathrm{~mm}$; DongBang Acupuncture 
Inc., Seoul, Korea) were inserted at a depth of 1-2 mm on each point. Needles were stimulated for 10 min using an EA system (ES-160; ITO Co. Ltd, Tokyo, Japan) with a low frequency $(10 \mathrm{~Hz})$ and low intensity (without muscle contractions). During EA stimulation, anaesthesia was provided using a rodent inhalation anaesthesia apparatus (Parkland Scientific, Coral Springs, FL, USA), which was equipped with interchangeable vaporizers for isoflurane (Hana Pharm. Co. Ltd., Hwasung, Korea). As carrier gas, $100 \%$ oxygen was used at a flow rate of $400 \mathrm{ml} / \mathrm{min}$. The anaesthetic gas was introduced into the nose mask through a thin tube. The concentration of anaesthetic gas in the nose mask and in the induction chamber was $2.8 \%$.

Measurement of plasma ghrelin and cholecystokinin levels. After sacrification, the collected plasma samples were promptly centrifuged at $4^{\circ} \mathrm{C}$ and the supernatant was stored at $-80^{\circ} \mathrm{C}$ until use. The plasma ghrelin and cholecystokinin (CCK) levels were determined using commercially available ELISA kits (cat. nos. EK-069-04 and MM-402, Mitsubishi Kagaku Iatron, Inc., Tokyo, Japan), according to the manufacturer's protocol.

Expression of c-Fos in the nucleus tractus solitarius (NTS). Rat brains were freshly dissected and brain stem tissue corresponding to the NTS cell group was obtained for analysis (20). For western blot analysis, tissues were homogenized in non-ionic detergent buffer $(150 \mathrm{mM} \mathrm{NaCl}, 1 \%$ Nonidet-P40, $1 \mathrm{mM}$ EDTA, 5\% glycerol and $25 \mathrm{mM}$ Tris-HCl, $\mathrm{pH} 7.5$ ) with protease inhibitor cocktail (Roche, Basel, Switzerland) for $20 \mathrm{~min}$ at $4^{\circ} \mathrm{C}$. Lysates were centrifuged at $21,130 \mathrm{x} \mathrm{g}$ for $40 \mathrm{~min}$ at $4^{\circ} \mathrm{C}$. Supernatant proteins were collected and quantified using the Bradford protein assay (Bio-Rad Laboratories, Inc., Hercules, CA, USA) and equal amounts of protein $(30 \mu \mathrm{g} /$ lane $)$ were separated via SDS-PAGE on a $8 \%$ gel. The separated proteins were transferred onto polyvinylidene difluoride membranes (cat. no. LC2002; Thermo Fisher Scientific, Inc., Waltham, MA, USA) and blocked with Tris buffered-saline containing Tween ${ }^{\mathrm{TM}} 20$ and $1 \%$ bovine serum albumin for $1 \mathrm{~h}$ at room temperature. The membranes were subsequently incubated with c-Fos primary antibody (1:500; cat. no. 2250) for $2 \mathrm{~h}$ at $25^{\circ} \mathrm{C}$, followed by incubation with horseradish peroxide (HRP)-conjugated secondary antibody (1:1,000; cat. no. 7074; both Cell Signaling Technology, Inc., Danvers, MA, USA) for $1 \mathrm{~h}$ at $25^{\circ} \mathrm{C}$. Protein bands were visualized using the Immobilon Western Chemiluminescent HRP Substrate (EMD Millipore, Billerica, MA, USA) and analyzed using a luminescent image analyzer (LAS-4000; GE Healthcare, Little Chalfont, UK). The relative intensities of each band were measured using ImageJ software (version 1.51n; National Institutes of Health, Bethesda, MD, USA). GAPDH (1:1,000; cat. no. 5174; Cell Signaling Technology, Inc.) was used as a loading control.

Measurement of 5-hydroxytryptamine (5-HT) levels in duodenal tissue and plasma. Rat duodenal tissue samples were homogenized by sonication in $0.4 \mathrm{M}$ perchloric acid. The homogenate was kept on ice for $20 \mathrm{~min}$ and then centrifuged at $21,130 \mathrm{x} \mathrm{g}$ for $20 \mathrm{~min}$ at $4^{\circ} \mathrm{C}$. Blood samples were obtained directly from the heart, added to a tube containing EDTA and centrifuged at $1,500 \mathrm{x}$ g for $10 \mathrm{~min}$ at $4^{\circ} \mathrm{C}$. Subsequently, $3.6 \mu \mathrm{l}$ of $70 \%$ perchloric acid was added to $100 \mu \mathrm{l}$ of a supernatant plasma sample. The mixture was centrifuged at 3,000 $\mathrm{x} g$ for $10 \mathrm{~min}$ at $4^{\circ} \mathrm{C}$. Supernatants were filtered through a $0.22-\mu \mathrm{m}$ filter and the concentrations of 5-HT were measured using high-performance liquid chromatography (HPLC) with electrochemical detection, as described previously (21). The mobile phase consisted of $85 \mathrm{mM}$ citrate, $100 \mathrm{mM}$ sodium acetate, $0.9 \mathrm{mM}$ sodium octyl sulfate, $0.2 \mathrm{mM}$ EDTA and $12 \%$ methanol, $\mathrm{pH} 3.7$. A total of $20 \mu \mathrm{l}$ was injected into an HPLC system fitted with a Nova-Pak C18 column $(60 \AA$; 3.9x150 mm; $4 \mu \mathrm{m}$; Waters Corporation, Milford, MA, USA) and electrochemical detector (2465; Waters Corporation), and the 515 HPLC pump (Waters Corporation) was operated at a constant flow rate of $1.0 \mathrm{ml} / \mathrm{min}$.

Reverse transcription-quantitative polymerase chain reaction $(R T-q P C R)$. Total RNA was extracted from the hypothalamus and gastric tissues using the Tri-RNA reagent (Favorgen, Kaohsiung, Taiwan), according to the manufacture's protocol. Complementary DNA (cDNA) was synthesized using the RevertAid First Strand cDNA Synthesis kit (Thermo Fisher Scientific, Inc.), according to the manufacturer's protocol. qPCR was subsequently performed using the PowerUp SYBR Green Master Mix (Thermo Fisher Scientific, Inc.), with primer pairs for each analyte gene (Table I). qPCR was performed using a Quantstudio 3 Real-Time PCR system (Thermo Fisher Scientific, Inc.) using the following thermocycling conditions: Initial denaturation at $95^{\circ} \mathrm{C}$ for $10 \mathrm{~min} ; 45$ cycles of $95^{\circ} \mathrm{C}$ for $3 \mathrm{sec}$, and the appropriate annealing temperature (Table I) for $30 \mathrm{sec}$. The relative mRNA expression levels were quantified using the $2^{-\Delta \Delta \mathrm{Cq}}$ method and normalized to the reference gene, $\beta$-actin (22).

Statistical analysis. All data, including body weight, food intake, water intake, urine excretion, levels of serum ghrelin, CCK, 5-HT and c-Fos expression were expressed as the mean \pm standard error of the mean which were normalized to the control group. Changes in body weight, food intake, water intake and urine excretion were compared prior to cisplatin injection and at days 1, 2, 3 and day 4 among the different acupuncture point stimulation groups, with positive values implying an increase in these parameters. As the experimental groups only included a small number of animals, a non-parametric analysis method was adopted for statistical analysis. For Experiment 1, the Kruskal-Wallis test was used for statistical analysis for each variable. Dunn's pairwise test with Bonferroni adjustment was performed as a post-hoc test. For the analysis of the results of Experiment 2, the Mann-Whitney U-test was used for comparison of each variable between the CV12 EA and control groups. The SPSS statistical package version 21 was used for all analyses (IBM Corp., Armonk, NY, USA). $\mathrm{P}<0.05$ was considered to indicate a statistically significant difference.

\section{Results}

Exploring the best conditions for the EA stimulation: Selection of the most effective acupuncture points. In Experiment 1, different acupuncture points were revealed to exert different anti-anorexigenic effects, as indicated by the body weight change and food intake of the animals. In the normal saline 
Table I. Primer pairs for reverse transcription-quantitative polymerase chain reaction.

\begin{tabular}{|c|c|c|}
\hline Gene & Primer sequence (5'-3') & Annealing temperature $\left({ }^{\circ} \mathrm{C}\right)$ \\
\hline \multirow[t]{2}{*}{ NGF } & F: TGACTCCAAGCACTGGAACTCAT & 60 \\
\hline & R: GTTTGTCGTCTGTTGTCAACGC & \\
\hline \multirow[t]{2}{*}{ BDNF } & F: GATGAGGACCAGAAGGTTCG & 65 \\
\hline & R: GATTGGGTAGTTCGGCATTG & \\
\hline \multirow[t]{2}{*}{ TNF- $\alpha$} & F: AAATGGGCTCCCTCTCATCAGTTC & 58 \\
\hline & R: TCTGCTTGGTGGTTTGCTACGAC & \\
\hline \multirow[t]{2}{*}{ IL-6 } & F: TCAACTCCATCTGCCCTTCAG & 58 \\
\hline & R: AAGGCAACTGGCTGGAAGTCT & \\
\hline \multirow[t]{2}{*}{ IL-1 $\beta$} & F: CACCTCTCAAGCAGAGCACAG & 60 \\
\hline & R: GGGTTCCATGGTGAAGTCAAC & \\
\hline \multirow[t]{2}{*}{ NPY } & F: TGTCTCAGGGCTGGATCTCT & 59 \\
\hline & R: TACTCCGCTCTGCGACACTA & \\
\hline \multirow[t]{2}{*}{ POMC } & F: GCTTCATGACCTCCGAGAAG & 59 \\
\hline & R: TCTTGATGATGGCGTTCTTG & \\
\hline \multirow[t]{2}{*}{ Ghrelin } & F: AGCCCAGCAGAGAAAGGAAT & 59 \\
\hline & R: GTGGCTGCAGTTTAGCTGGT & \\
\hline \multirow[t]{2}{*}{$\beta$-actin } & F: AАGТСССТСАСССТСССАAАAG & 58 \\
\hline & R: AAGCAATGCTGTCACCTTCCC & \\
\hline
\end{tabular}

NGF, nerve growth factor; BDNF, brain-derived neurotrophic factor; TNF- $\alpha$, tumor necrosis factor $\alpha$; IL-6, interleukin 6; IL-1 $\beta$, interleukin $1 \beta$; NPY, neuropeptide Y; and POMC, proopiomelanocortin.

group (untreated control), a progressive increase of food intake was observed during the study. However, after cisplatin injection, the food intake decreased rapidly in the EA groups and the Cisplatin only group of rats. The decrease of food intake was comparatively lower in the CV12 EA group between days 0 and 1 (Normal control, $2.2 \pm 2.3 \mathrm{~g}$; Cisplatin group, $-1.1 \pm 1.8 \mathrm{~g}$; PC6, -4.2 \pm 4.4 g; ST36, $-1.6 \pm 2.0 \mathrm{~g} ; \mathrm{CV} 12,0.0 \pm 2.7 ; \mathrm{P}=0.648$ ). On days 2 and 3 , the lowest decrease in food intake among the EA groups was observed in the CV12 EA group and there was a statistically significant difference (day 2 vs. day 0: Normal

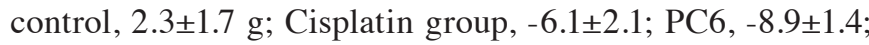
ST36, $-7.3 \pm 1.8 \mathrm{~g} ; \mathrm{CV} 12,-6.3 \pm 0.7 \mathrm{~g} ; \mathrm{P}=0.032$. Day 3 vs. day 0 : Normal control, $3.1 \pm 2.5 \mathrm{~g}$; Cisplatin group, $-10.9 \pm 1.9 \mathrm{~g}$; PC6, $-9.2 \pm 2.7 \mathrm{~g} ;$ ST36, -9.1 $\pm 2.6 \mathrm{~g}$; CV12, $-4.9 \pm 2.0 \mathrm{~g} ; \mathrm{P}=0.019)$. At the final assessment on day 4 , the CV12 group also exhibited the lowest decrease in food intake compared with that at day 0 among the EA groups, but there was no significant difference (Normal control, 3.6 \pm 1.7 ; Cisplatin group, -9.3 \pm 3.7 g; PC6, $-10.8 \pm 3.4$ g; ST36; $-10.0 \pm 3.8$ g; CV12, $-6.8 \pm 2.7 ; \mathrm{P}=0.084)$.

As displayed in Fig. 1, the body weight increased significantly in the Normal control group between Day 0 and 2 and Day 0 and 3, however, there was an overall decrease in body weight following treatment with Cisplatin. In all of the EA groups, the body weight increased gradually, but the CV12 group exhibited the highest increase in most assessments (day 1 vs. 0: Normal control, 2.9 $\pm 6.6 \mathrm{~g}$; Cisplatin group, 5.9 \pm 0.7 ;

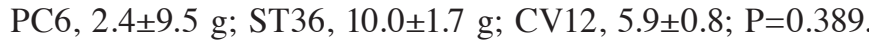
Day 2 vs. 0: Normal control, $15.1 \pm 2.1 \mathrm{~g}$; Cisplatin group, $7.2 \pm 3.1$ g; PC6, 5.6 \pm 7.1 g; ST36, 8.3 vs. 2.9 ; CV12, 7.5 \pm 0.23 g $\mathrm{P}=0.223$. Day 3 vs. 0: Normal control, 22.9 $\pm 1.6 \mathrm{~g}$; Cisplatin

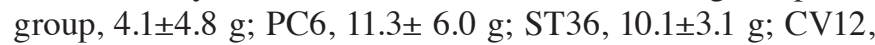

$13.8 \pm 1.8 \mathrm{~g} ; \mathrm{P}=0.63$. Day 4 vs. 0: Normal control, $28.6 \pm 2.0 \mathrm{~g}$;

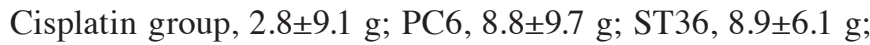
CV12, 14.0 $\pm 4.9 ; \mathrm{P}=0.156$ ). Regarding the water intake and urine excretion, no significant differences were identified between any of the groups (Table II).

Experiment 2: Effect of EA at CV12 on the serum ghrelin and $C C K$ levels in rats with cisplatin-induced anorexia. According to Experiment 1, food intake decreased the least in rats treated by EA at CV12 with an inhibition of normal weight increase in cisplatin-induced anorexia compared with the other acupuncture point stimulation groups (Table II). Therefore, the CV12 acupoint was used to assess the effect of EA on the regulation of peptides that are closely associated with appetite. An increased level of serum ghrelin was observed in the CV12 EA group compared with that in the control group at day 3 $(65.6 \pm 0.2$ vs. $46.4 \pm 0.1 \mathrm{pg} / \mathrm{ml} ; \mathrm{P}=0.057)$, and $\mathrm{CCK}$ exhibited a similar pattern $(706.2 \pm 24.7$ vs. $547.6 \pm 21.4 \mathrm{pg} / \mathrm{ml} ; \mathrm{P}=0.057$; Fig. 2). In addition, RT-qPCR analysis indicated that the expression of ghrelin mRNA in the stomach was significantly increased in the CV12 EA group compared with that in the control group (Fig. 3).

Duodenal and plasma 5-HT concentration. Regarding the secretion of duodenal 5-HT in rats, the levels were undetectably low in the duodenum, which suggests that the 5-HT concentration in the duodenum was not influenced by the CV12 EA treatment. In addition, the plasma levels of 5-HT were higher in the control group than that in the CV12 EA group, but the difference was not statistically significant (207.3 \pm 70.1 vs. $66.3 \pm 33.4$ ng/ml; $P=0.132$; Fig. 4). 

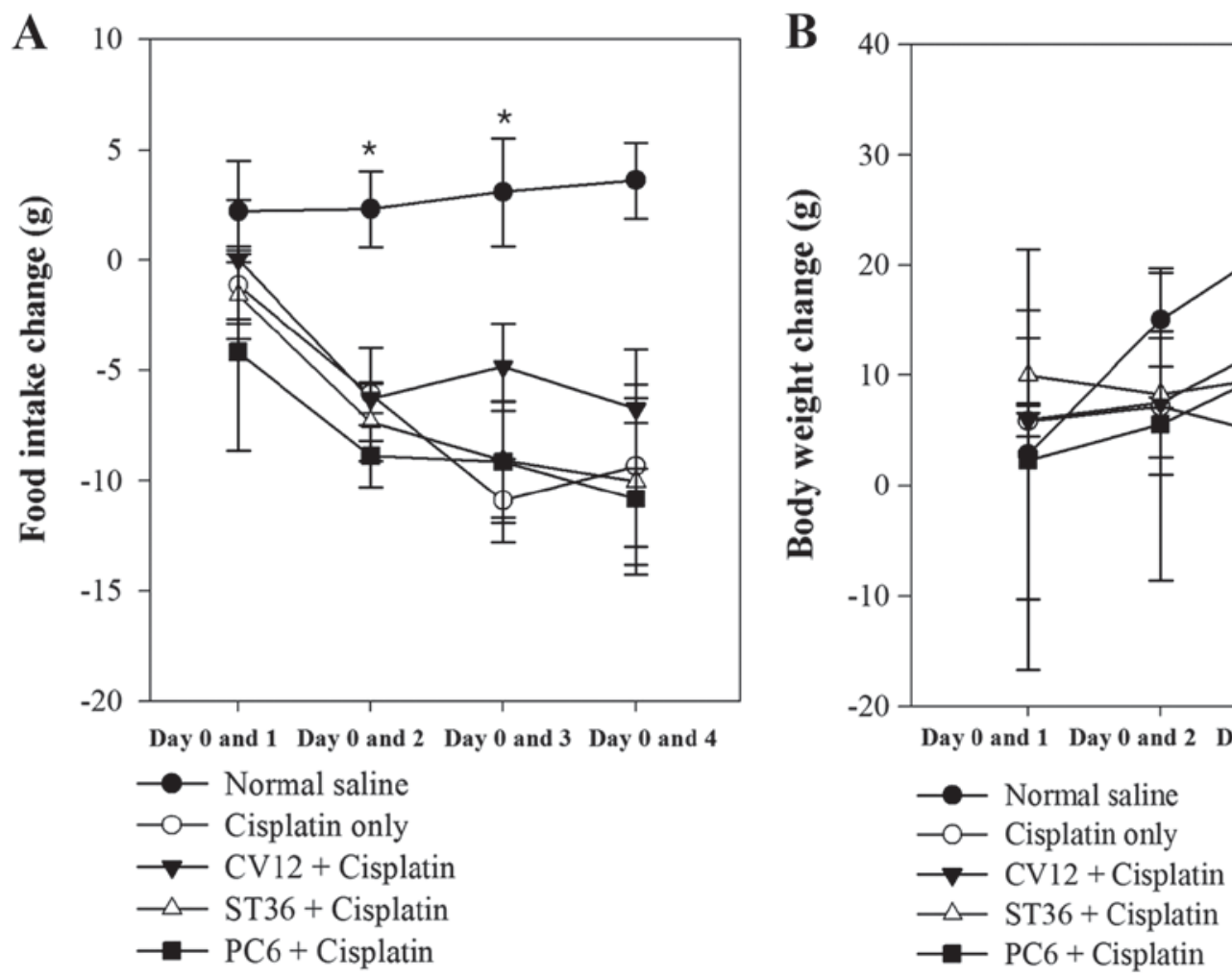

Figure 1. Food intake and body weight changes in experiment 1 . Changes in (A) food intake and (B) body weight at days 1,2, 3 and 4 vs. day 0 (prior to cisplatin injection) were compared among 5 groups and positive values indicate an increase in food intake and body weight vs. baseline. " $\mathrm{P}<0.05$.

$\mathbf{A}$

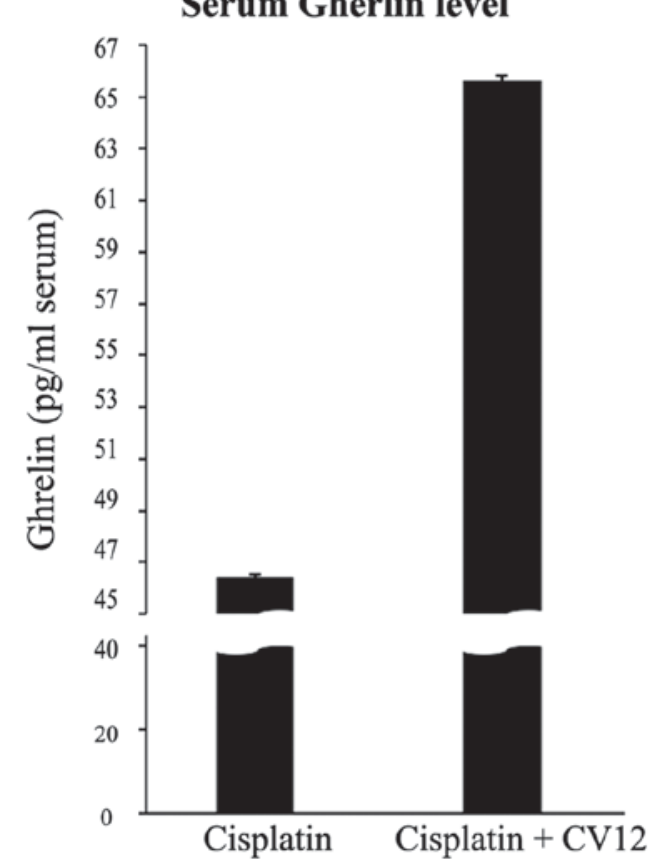

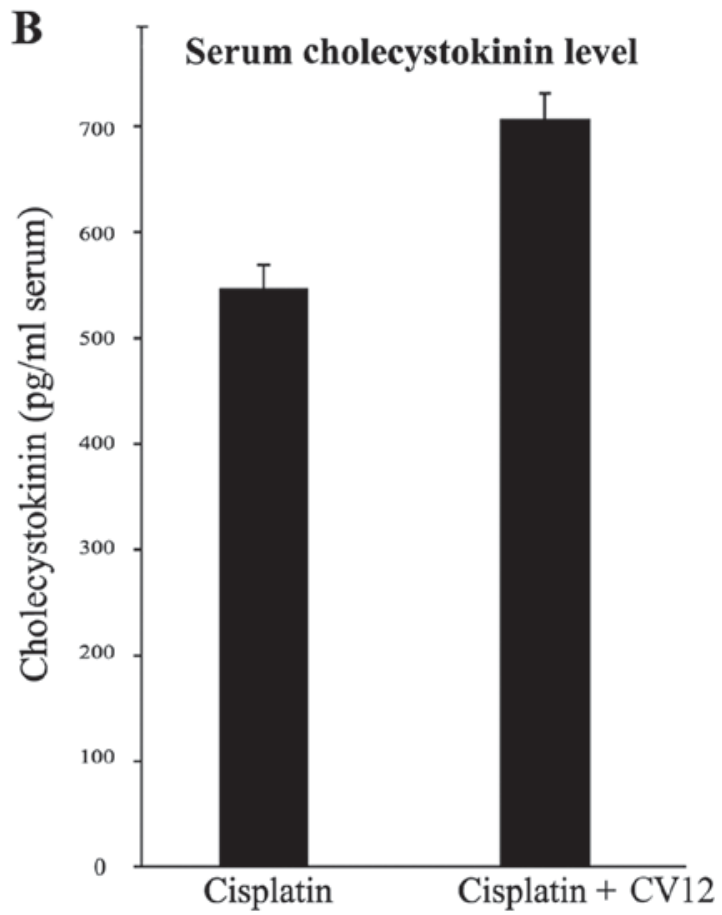

Figure 2. Serum ghrelin and cholecystokinin levels. Serum ghrelin (A) and cholecystokinin (B) levels were compared between the Cisplatin + CV12 group and the Cisplatin group. The Mann-Whitney U-test was used for comparison. Groups: Cisplatin, control group in the cisplatin injection-induced anorexia rat model; Cisplatin + CV12, CV12 electroacupuncture group in the cisplatin injection-induced anorexia rat model.

mRNA expression of neurotropic factors and cytokines in hypothalamus. Although there was no statistical significance, the expression of NPY mRNA in the hypothalamus was increased in the cisplatin + CV12 EA group compared with that in the cisplatin group. However, the expression of POMC mRNA in the hypothalamus was not significantly affected by CV12 EA 
Table II. Changes in food intake, body weight, water intake and urine excretion in Experiment 1 compared with day 0.

\begin{tabular}{|c|c|c|c|c|}
\hline Parameter/group & Day 1 & Day 2 & Day 3 & Day 4 \\
\hline \multicolumn{5}{|c|}{ Changes in food intake (g) } \\
\hline Normal control & $2.2 \pm 2.3$ & $2.3 \pm 1.7$ & $3.1 \pm 2.5$ & $3.6 \pm 1.7$ \\
\hline Cisplatin & $-1.1 \pm 1.8$ & $-6.1 \pm 2.1$ & $-10.9 \pm 1.9^{a}$ & $-9.3 \pm 3.7$ \\
\hline CV12 EA & $0 \pm 2.7$ & $-6.3 \pm 0.7$ & $-4.9 \pm 2.0$ & $-6.8 \pm 2.7$ \\
\hline ST36 EA & $-1.6 \pm 2.0$ & $-7.3 \pm 1.8$ & $-9.1 \pm 2.6$ & $-10.0 \pm 3.8$ \\
\hline PC6 EA & $-4.2 \pm 4.4$ & $-8.9 \pm 1.4$ & $-9.2 \pm 5.5$ & $-10.8 \pm 3.4$ \\
\hline P-value & 0.648 & 0.032 & 0.019 & 0.084 \\
\hline \multicolumn{5}{|c|}{ Changes in body weight (g) } \\
\hline Normal control & $2.8 \pm 6.6$ & $15.0 \pm 2.1$ & $22.9 \pm 1.6$ & $28.6 \pm 2.0$ \\
\hline Cisplatin & $5.9 \pm 6.9$ & $7.2 \pm 3.1$ & $4.1 \pm 4.8$ & $2.8 \pm 9.1$ \\
\hline CV12 EA & $5.6 \pm 0.8$ & $7.5 \pm 0.2$ & $13.8 \pm 1.8$ & $14.0 \pm 4.9$ \\
\hline ST36 EA & $10.0 \pm 1.7$ & $8.3 \pm 2.9$ & $10.1 \pm 3.1$ & $8.9 \pm 6.1$ \\
\hline PC6 EA & $2.4 \pm 9.5$ & $5.6 \pm 7.1$ & $11.3 \pm 6.0$ & $8.8 \pm 9.7$ \\
\hline P-value & 0.389 & 0.223 & 0.063 & 0.156 \\
\hline \multicolumn{5}{|c|}{ Changes in water intake (ml) } \\
\hline Normal control & $-9.8 \pm 8.1$ & $12.0 \pm 6.7$ & $-3.5 \pm 7.1$ & $-5.5 \pm 5.2$ \\
\hline Cisplatin & $3.0 \pm 4.0$ & $-6.5 \pm 6.7$ & $-11.5 \pm 3.4$ & $-1.5 \pm 5.9$ \\
\hline CV12 EA & $3.8 \pm 6.8$ & $4.0 \pm 4.8$ & $3.0 \pm 5.6$ & $3.5 \pm 5.6$ \\
\hline ST36 EA & $-0.3 \pm 5.0$ & $-2.5 \pm 10.3$ & $-7.0 \pm 1.7$ & $-8.5 \pm 2.8$ \\
\hline PC6 EA & $1.0 \pm 9.4$ & $-10.0 \pm 4.5$ & $1.5 \pm 7.6$ & $-9.0 \pm 4.0$ \\
\hline P-value & 0.797 & 0.154 & 0.247 & 0.289 \\
\hline \multicolumn{5}{|c|}{ Changes in urine excretion (ml) } \\
\hline Normal control & $-2.6 \pm 1.2$ & $-2.6 \pm 1.2$ & $-2.9 \pm 1.1$ & $-1.1 \pm 1.4$ \\
\hline Cisplatin & $-2.0 \pm 1.8$ & $-2.0 \pm 1.8$ & $-3.4 \pm 1.5$ & $-1.1 \pm 1.7$ \\
\hline CV12 EA & $0.0 \pm 0.0$ & $0.0 \pm 0.0$ & $0.5 \pm 2.8$ & $0.8 \pm 3.4$ \\
\hline ST36 EA & $-0.6 \pm 0.4$ & $-0.6 \pm 0.4$ & $-1.2 \pm 2.1$ & $3.5 \pm 2.5$ \\
\hline PC6 EA & $-2.4 \pm 0.7$ & $-2.4 \pm 0.7$ & $3.0 \pm 2.8$ & $3.1 \pm 1.9$ \\
\hline P-value & 0.213 & 0.213 & 0.316 & 0.363 \\
\hline
\end{tabular}

Values are presented as the mean \pm standard error of the mean. All data were analyzed using the Kruskal-Wallis test followed by Dunn's pairwise test with Bonferroni adjustment. ${ }^{a} \mathrm{P}<0.05$ vs. normal control group. EA, electroacupuncture.

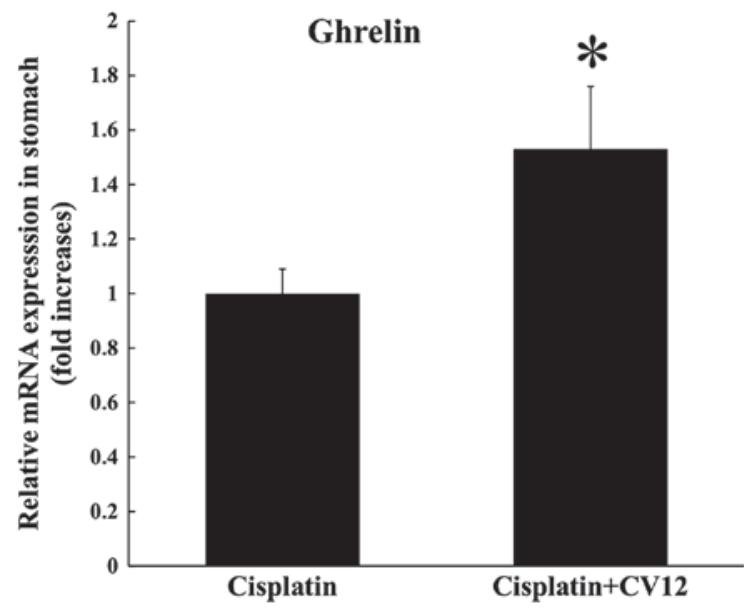

Figure 3. mRNA expression of ghrelin in stomach tissue. The mRNA expression of ghrelin in stomach tissue was compared between the Cisplatin + CV12 group and the Cisplatin group. The Mann-Whitney U-test was used for comparison. Groups: Cisplatin, control group in the cisplatin injection-induced anorexia rat model; Cisplatin + CV12, CV12 electroacupuncture group in the cisplatin injection-induced anorexia rat model. ${ }^{*} \mathrm{P}<0.05$. compared with that in the cisplatin group. Furthermore, the results indicated that the mRNA expression levels of BDNF in the CV12 EA group were significantly higher compared with those in the cisplatin only group, whereas there were no changes in the mRNA expression levels of NGF. Furthermore, the mRNA expression levels of cytokines, including TNF- $\alpha$, IL-1 $\beta$ and IL-6, were not significantly different between the two groups (Fig. 5).

c-Fos expression in the NTS. Western blot analysis indicated that in the CV12 EA group, the protein expression levels of c-Fos in the NTS were downregulated compared with those in the control group; however, this reduction was not statistically significant (Fig. 6).

\section{Discussion}

In the present study, the anti-anorexigenic effect of EA was evaluated in a rat model of cisplatin-induced anorexia. Among the potential acupuncture points, including CV12, PC6, and 

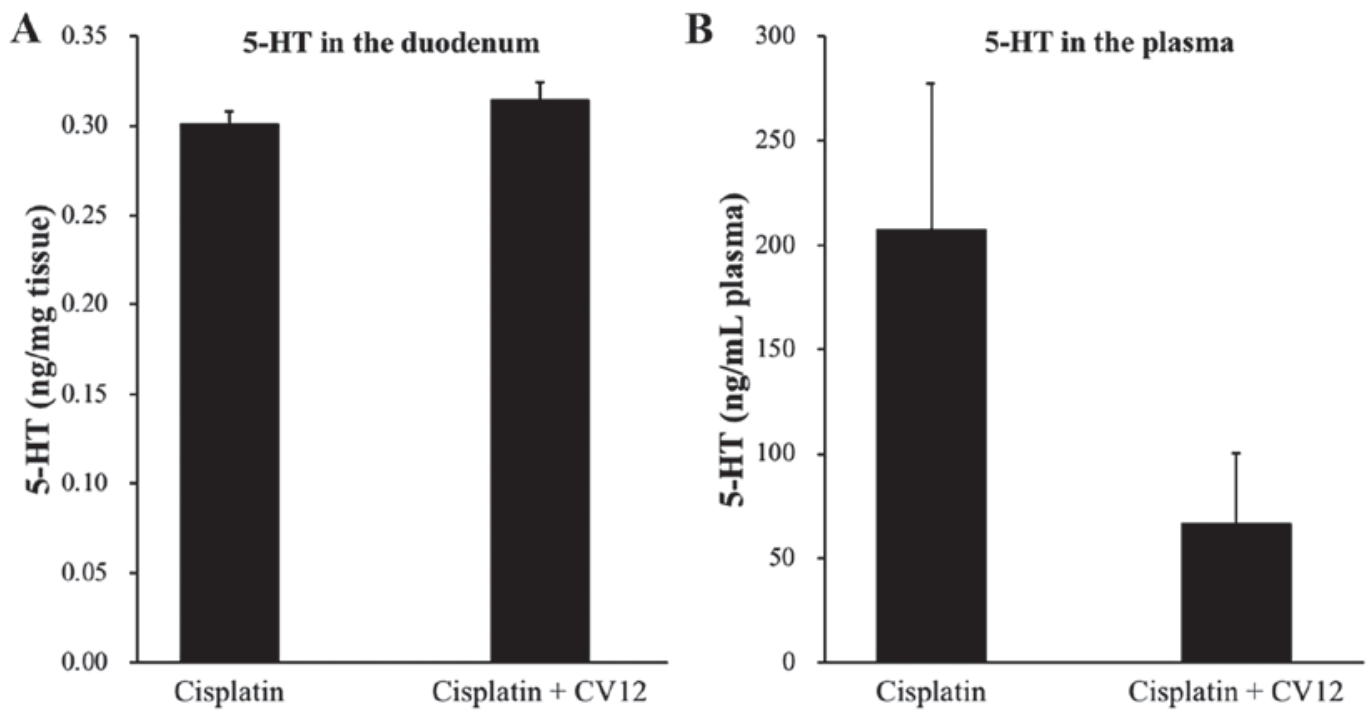

Figure 4. Concentration of 5-HT measured using high-performance liquid chromatography in (A) the duodenum and (B) plasma. The levels of 5-HT in the duodenum and plasma were compared between the Cisplatin + CV12 group and the Cisplatin group. The Mann-Whitney U-test was used for comparison. Groups: Cisplatin, control group in the cisplatin injection-induced anorexia rat model; Cisplatin + CV12, CV12 electroacupuncture group in the cisplatin injection-induced anorexia rat model. 5-HT, 5-hydroxytryptamine.

ST36, CV12 was observed to be the most effective in preventing the cisplatin-associated decrease in food intake and inhibition of body weight increase. Assessment of the serum levels of ghrelin and CCK revealed that compared with the controls, EA at CV12 enhanced the secretion of ghrelin and CCK into the serum. Consistent with these results, the mRNA expression of ghrelin in the stomach was also increased by EA at CV12. These results suggest that EA treatment at CV12 may reduce CIA through increasing the secretion of anti-anorexigenic peptides, including ghrelin and CCK.

The present study has several advantages. First, the point-specific effects of acupuncture were compared to reveal the most effective stimulation points of EA. A total of 361 standard acupuncture points and numerous Ashi-points are currently used in clinical practice (23), and each point is selected based on the location and point-specific effect of acupuncture (24). CV12, PC6 and ST36 are generally regarded as effective acupuncture points for treating GI symptoms and diseases $(25,26)$. In addition to being used for treating various gastric symptoms, these points are used to improve appetite in various populations $(27,28)$. In the present study, these possible acupuncture points were tested and the effect of EA at these points on promoting appetite and inhibiting weight loss were compared in a rat model of cisplatin-induced anorexia. The present results were consistent with those of a previous study (12). Furthermore, the underlying mechanisms of the effects of EA on CIA were assessed. In common clinical practice, 5-HT receptor antagonists are the most commonly prescribed drugs for the prevention of CINV in cancer patients (29). Previous clinical (30) and experimental studies (12) have typically focused on the anti-emetic effect of acupuncture during chemotherapy in subjects with cancer. 5-HT secretion and 5-HT receptor regulation, which are key factors in nausea and vomiting, have also been a major focus of acupuncture research (12). In the present study, the serum levels of ghrelin and CCK, which are closely associated with appetite, were evaluated, and it was revealed that EA may increase the secretion of ghrelin and CCK in rats with cisplatin-induced anorexia. EA also increased the expression of ghrelin mRNA in stomach tissue of cisplatin-induced anorexic rats. It may be hypothesized that EA exerts its effects to reduce the problems in cancer patients undergoing chemotherapy associated with intake of nutrition via multiple pathways. Finally, the long-term effect of acupuncture ( $>2$ days) was assessed, which was not performed in the previous study (12). The present study indicated that cisplatin significantly reduced the food intake and the increase in body weight at $72 \mathrm{~h}$ after administration. To assess the subacute or chronic anorexia associated with chemotherapy, longer-term studies of $>1$ week may be necessary.

The present study also has several disadvantages that should be addressed. First, short evaluation periods led to significant setbacks due to lack of information on the long-term effects of treatments, and in addition, the number of rats was small. Furthermore, CINV has been a major focus of research on chemotherapy, and thus, numerous studies have evaluated nausea and vomiting in animal models. Pica behavior is observed in animal models that experience environment- or therapy-triggered nausea (31). Taking notice of this phenomenon, researchers have begun to evaluate kaolin intake during chemotherapy instead of nausea and vomiting in rats (32). In the present study, kaolin intake was not assessed for evaluating nausea and vomiting in rats, as this may rather be considered to be an alternative approach to directly assess nausea and vomiting. In addition, the assessment of kaolin intake may prohibit the precise measurement of food intake due to the consumption of kaolin at the same time as food. Furthermore, while a previous study assessed the effect of multi-point stimulation (12), this was not assessed in the present study. Acupuncture is generally performed on multiple points at the same time. There are numerous significant differences between single-point and multi-point stimulation, 

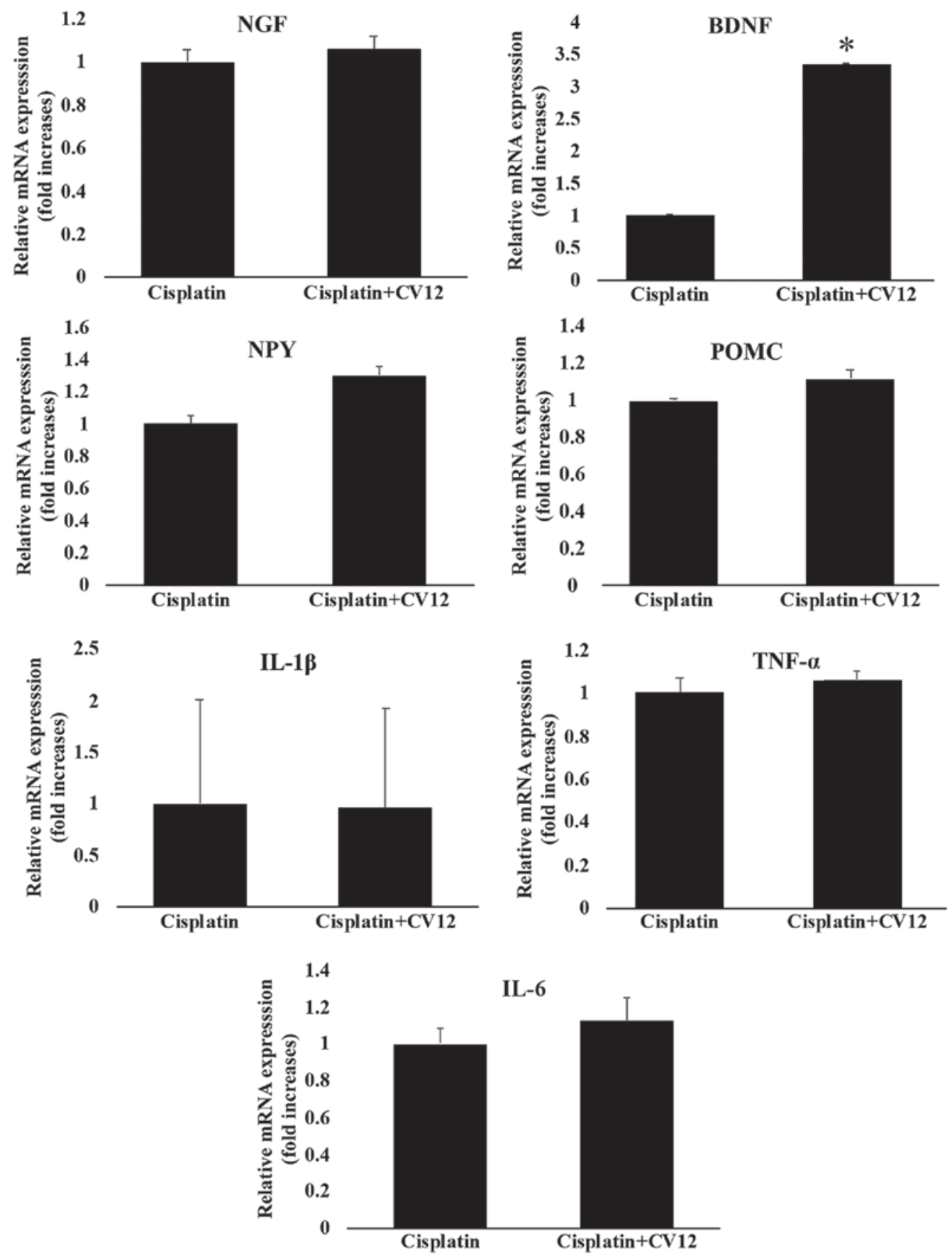

Figure 5. mRNA expression of neurotropic factors and cytokines in the hypothalamus. The mRNA expression of neurotropic factors and cytokines in the hypothalamus, including NGF, BDNF, TNF- $\alpha$, IL-6, IL-1 $\beta$, NPY and POMC were compared between the Cisplatin + CV12 group and the Cisplatin group. The Mann-Whitney U-test was used for comparison. Groups: Cisplatin, control group in the cisplatin injection-induced anorexia rat model; Cisplatin + CV12, CV12 electroacupuncture group in the cisplatin injection-induced anorexia rat model. NGF, nerve growth factor; BDNF, brain-derived neurotrophic factor; TNF- $\alpha$, tumor necrosis factor $\alpha$; IL-6, interleukin 6; NPY, neuropeptide Y; POMC, pro-opiomelanocortin. ${ }^{*} \mathrm{P}<0.05$.

including the aspect of the location of the stimulation and the intensity; this may affect the possible mechanism of the effects of acupuncture. To better reflect the situation in clinical practice, a combination of different acupuncture stimulation points should be evaluated in future studies. In addition, the present study did not include any group where rats were only kept without injection of normal saline and acupuncture stimulation in order to evaluate whether single acupuncture may influence food consumption or weight changes. Instead, we included groups treated with cisplatin only and sham acupuncture at non-acupuncture points as control groups to show the potential effects of acupuncture. Future study is required with inclusion of such a group for assessing the effect of acupuncture stimulation on the feeding behaviour. In addition to this, EA at a non-acupuncture point is also necessary to evaluate whether there is an acupuncture point specific effect or not. Finally, most comparisons of the present study did not indicate any statistically significant differences between the groups, which may originate from the small sample size per each group and the inappropriate experimental conditions, including the 

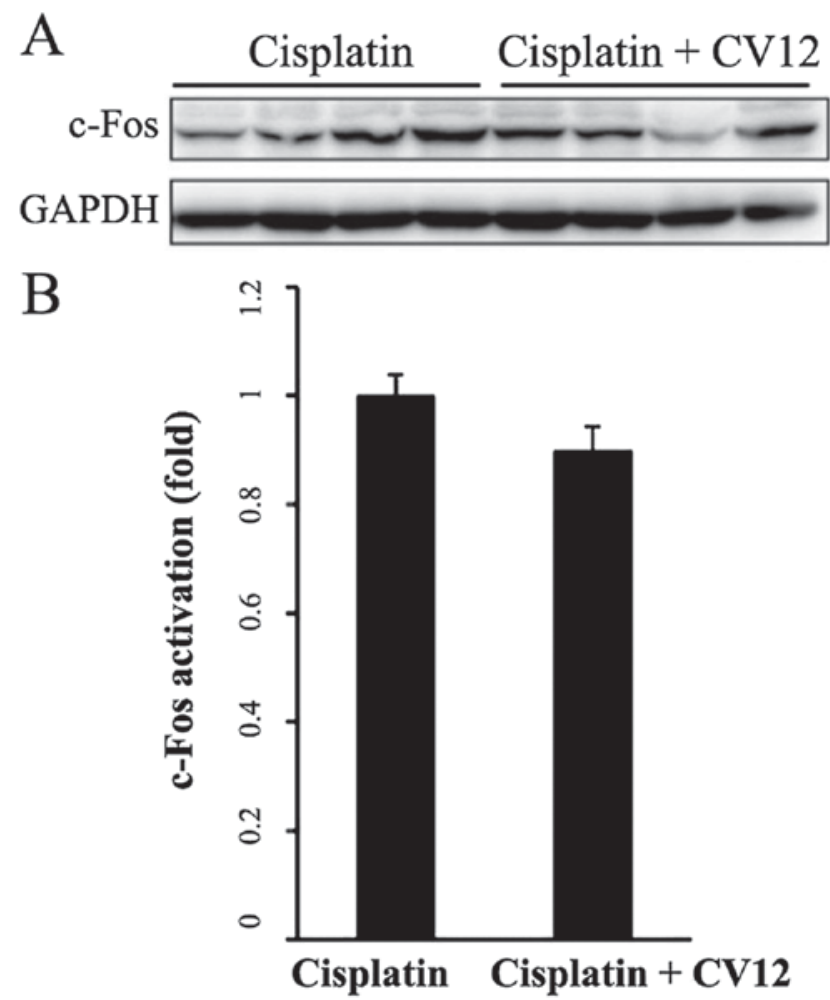

Figure 6. Western blot analysis of c-Fos protein in the brainstem nucleus of the solitary tract area. (A) A representative western blot image and (B) the quantitative results are presented. C-Fos protein levels in the brainstem nucleus of the solitary tract were compared between the Cisplatin + CV12 group and the Cisplatin group. The Mann-Whitney U-test was used for comparison. Groups: Cisplatin, control group in the cisplatin injection-induced anorexia rat model; Cisplatin + CV12, CV12 electroacupuncture group in the cisplatin injection-induced anorexia rat model.

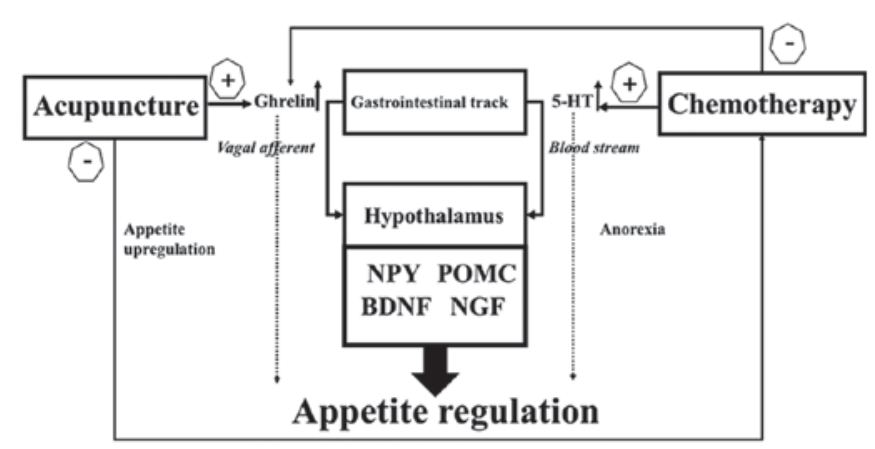

Figure 7. Mechanism of chemotherapy-induced anorexia prevention by electroacupuncture. NPY, neuropeptide-Y; POMC, pro-opiomelanocortin; BDNF, brain-derived neurotrophic factor; NGF, nerve growth factor; 5-HT, 5-hydroxytryptamine.

frequency or intensity of EA stimulation; indeed, these remain unsolved issues urging future research.

The present study suggests that the mechanisms by which acupuncture ameliorates CIA and CINV include the regulation of 5-HT and CNS activation. Cisplatin and other chemotherapeutic agents induce a mucosal injury of the intestines that includes serotonin-producing cells (33), and induce nausea and vomiting through signal transmission to the brain emetic center via the vagus nerve to abruptly release excessive 5-HT (34). Based on the results of the present and a previous study (12), EA at CV12 may affect 5-HT secretion in the duodenum and plasma, and deactivate neuronal transmission in the NTS, which may be evaluated through c-Fos protein expression as a metabolic marker of neuronal activation $(35,36)$. These results suggest that acupuncture may exert an anti-emetic effect through regulating the secretion of the nausea-triggering peptide 5-HT and controlling the emetic center in the NTS through afferent vagal stimulation.

NPY and POMC, which are abundant in hypothalamic arcuate nucleus, have been reported to engage in the promotion or inhibition of feeding, respectively (37). In the present study, the mRNA expression of these two genes in the hypothalamus was evaluated. It was indicated that EA-mediated appetite regulation is not associated with the mRNA expression of NPY and POMC. BDNF has been reported to be highly expressed in the ventromedial hypothalamus, where it regulates energy homeostasis $(37,38)$. In the present study, the mRNA expression of BDNF exhibited a significant increase compared with that in the control group. Previously, glucose administration under fasting conditions increased in the mRNA expression levels of BDNF consistently with its role in satiety (38). Furthermore, no changes in the mRNA expression levels of cytokines, including TNF- $\alpha$, IL- 6 and IL-1 $\beta$, were detected in the hypothalamus. Therefore, the present results suggest that the EA-mediated increase of BDNF mRNA expression in the hypothalamus may be a crucial mechanism for the regulation of CIA, but the exact mechanism involving NPY, POMC, NGF and BDNF should be further evaluated in the future.

In the present study, chemotherapy-induced eating anorexia and loss of appetite were examined. It was indicated that acupuncture stimulation increased weight gain and food intake in an animal model of CIA, and the present study reproduced the results observed in a previous study (12). Ghrelin is a multi-functional endogenous peptide that is important for maintaining energy metabolism, and that affects the eating behavior (36). CCK is secreted in the duodenum for fat and protein digestion, and typically acts as a hunger suppressor through inducing satiety and reducing food intake (39). Plasma ghrelin levels are decreased after cisplatin injection, which may account for the loss of appetite in chemotherapy patients (40). However, little is known about the effect of cisplatin injection on CCK levels. Serum ghrelin levels were increased after CV12 EA treatment (12), and a similar increase in CCK levels was also observed in the present study. These results suggest that the beneficial effect of CV12 EA on cisplatin-induced anorexia is mediated via humoral appetite regulation through central mechanisms involving POMC and NPY as well as peripheral mechanisms involving 5-HT, ghrelin and CCK as illustrated in Fig. 7. In the future, more research is required to elucidate the mechanisms underlying the effect of acupuncture.

In conclusion, CV12 EA may improve the appetite by increasing the secretion of serum ghrelin and CCK, in addition to exerting an anti-emetic effect through regulation of 5-HT secretion and vagal nerve activation in cisplatin-induced anorexic rats. Nutrition-associated problems caused by chemotherapy require to be addressed using a multidirectional approach, and acupuncture may be considered as one of the potential complementary therapies for this condition. 


\section{Acknowledgements}

Not applicable.

\section{Funding}

The current study was supported by a grant from the Korea Health Technology R\&D Project through the Korea Health Industry Development Institute, funded by the Ministry of Health \& Welfare, Republic of Korea (grant no. HI15C0089).

\section{Availability of data and materials}

All data generated or analyzed during this study are included in this published article.

\section{Authors' contributions}

KSK, JWK and THK contributed to the concept and design of the study. KSK, WH, YB, HJC, JYB, JHS, JWK and THK performed the experiments. KSK, JWK and THK analyzed the data. KSK and THK prepared the first draft and revised the manuscript. All authors read and approved the final manuscript.

\section{Ethical approval and consent to participate}

All procedures involving the use of live animals described in the present study were approved by the Institutional Animal Care and Use Committee of Gachon University (Seongnam, Korea; approval no. GIACUC-R2015011) in December 2015. In addition, animal experimentation was performed in strict accordance with the National Institutes of Health Guide for the Care and Use of Experimental Animals.

\section{Patient consent for publication}

Not applicable.

\section{Competing interests}

The authors declare that they have no competing interests.

\section{References}

1. Wie GA, Cho YA, Kim SY, Kim SM, Bae JM and Joung H Prevalence and risk factors of malnutrition among cancer patients according to tumor location and stage in the national cancer center in Korea. Nutrition 26: 263-268, 2010

2. Pressoir M, Desné S, Berchery D, Rossignol G, Poiree B, Meslier M, Traversier S, Vittot M, Simon M, Gekiere JP, et al: Prevalence, risk factors and clinical implications of malnutrition in french comprehensive cancer centres. Br J Cancer 102: 966-971, 2010

3. Segura A, Pardo J, Jara C, Zugazabeitia L, Carulla J, de Las Peñas R, García-Cabrera E, Luz Azuara M, Casadó J and Gómez-Candela C: An epidemiological evaluation of the prevalence of malnutrition in Spanish patients with locally advanced or metastatic cancer. Clin Nutr 24: 801-814, 2005.

4. Pender A, Moocraft SY and Lee DLY: Side effects and complications of cancer and its treatment. In: Clinical Problems in Oncology: A Practical Guide to Management. 1st edition. Wiley-Blackwell, Chichester, UK, pp277-286, 2014.

5. von Meyenfeldt M: Cancer-associated malnutrition: An introduction. Eur J Oncol Nurs 9 (Suppl 9): S35-S38, 2005.
6. Navari RM: Pharmacological management of chemotherapyinduced nausea and vomiting. Focus on recent developments. Drugs 69: 515-533, 2009.

7. Chen YP, Liu T, Peng YY, Wang YP, Chen H, Fan YF and Zhang L: Acupuncture for hot flashes in women with breast cancer: A systematic review. J Cancer Res Ther 12: 535-542, 2016.

8. Chiu HY, Hsieh YJ and Tsai PS: Systematic review and meta-analysis of acupuncture to reduce cancer-related pain. Eur J Cancer Care (Engl) 26: 12457, 2017.

9. Zeng Y, Luo T, Finnegan-John J and Cheng AS: Meta-analysis of randomized controlled trials of acupuncture for cancer-related fatigue. Integr Cancer Ther 13: 193-200, 2014.

10. Lu W, Hu D, Dean-Clower E, Doherty-Gilman A, Legedza AT, Lee H, Matulonis U and Rosenthal DS: Acupuncture for chemotherapy-induced leukopenia: Exploratory meta-analysis of randomized controlled trials. J Soc Integr Oncol 5: 1-10, 2007.

11. Wu X, Chung VC, Hui EP, Ziea ET, Ng BF, Ho RS, Tsoi KK, Wong SY and Wu JC: Effectiveness of acupuncture and related therapies for palliative care of cancer: Overview of systematic reviews. Sci Rep 5: 16776, 2015.

12. Cui Y, Wang L, Shi G, Liu L, Pei P and Guo J: Electroacupuncture alleviates cisplatin-induced nausea in rats. Acupunct Med 34: 120-126, 2016.

13. Kim TH, Kang JW and Lee MS: What is lost in the acupuncture trial when using a sham intervention? Acupunct Med 35: 384-386, 2017.

14. Jeon JH, Yoon J, Cho CK, Jung IC, Kim S, Lee SH and Yoo HS: Effect of acupuncture for radioactive-iodine-induced anorexia in thyroid cancer patients: A randomized, double-blinded, sham-controlled pilot study. Integr Cancer Ther 14: 221-230, 2015.

15. Yoon SL, Grundmann O, Williams JJ and Carriere G: Novel intervention with acupuncture for anorexia and cachexia in patients with gastrointestinal tract cancers: A feasibility study. Oncol Nurs Forum 42: E102-E109, 2015.

16. Date Y, Toshinai K, Koda S, Miyazato M, Shimbara T, Tsuruta T, Niijima A, Kangawa $\mathrm{K}$ and Nakazato M: Peripheral interaction of ghrelin with cholecystokinin on feeding regulation. Endocrinology 146: 3518-3525, 2005.

17. Ecelbarger CA, Sands JM, Doran JJ, Cacini W and Kishore BK: Expression of salt and urea transporters in rat kidney during cisplatin-induced polyuria. Kidney Int 60: 2274-2282, 2001.

18. Borner T, Loi L, Pietra C, Giuliano C, Lutz TA and Riediger T: The ghrelin receptor agonist HM01 mimics the neuronal effects of ghrelin in the arcuate nucleus and attenuates anorexia-cachexia syndrome in tumor-bearing rats. Am J Physiol Regul Integr Comp Physiol 311: R89-R96, 2016.

19. Li Z: Experimental acupuncture science. Beijing: China Press of Traditional Chinese Medicine 327: 146, 2003.

20. Zhou D, Wan Y, Xie D, Wang Y, Wei J, Yan Q, Lu P, Mo L, Xie J, Yang S and Qi X: DNMT1 mediates chemosensitivity by reducing methylation of miRNA-20a promoter in glioma cells. Exp Mol Med 47: e182, 2015.

21. Tianzhu Z, Shihai Y and Juan D: Antidepressant-like effects of cordycepin in a mice model of chronic unpredictable mild stress. Evid Based Complement Alternat Med 2014: 43856, 2014.

22. Livak KJ and Schmittgen TD: Analysis of relative gene expression data using real-time quantitative PCR and the 2(-Delta Delta C(T)) method. Methods 25: 402-408, 2001.

23. WHO standard acupuncture point locations in the Western Pacific 106 Region. World Health Organization, Geneva, pp67, $158,229,2008$.

24. Choi EM, Jiang F and Longhurst JC: Point specificity in acupuncture. Chin Med 7: 4, 2012.

25. Takahashi T: Acupuncture for functional gastrointestinal disorders. J Gastroenterol 41: 408-417, 2006.

26. Jinsheng H: Acupuncture treatment of vomiting. J Traditional Chin Med 28: 75-78, 2008 (In Chinese).

27. Kim HY, Seong WY and Kim KB: A literature study on treatment of infantile anorexia based on Chinese medical journals J Korean Oriental Pediatrics 27: 87-98, 2013.

28. Yoveline A, Abdullah M, Darmawan G, Mihardja H and Sungkar S: Acupuncture in the management of functional dyspepsia. The Indonesian Journal of Gastroenterology, Hepatology, and Digestive Endoscopy 13: 49-55, 2012. https:// media.neliti.com/media/publications/67584-ID-none.pdf.

29. Billio A, Morello E and Clarke MJ: Serotonin receptor antagonists for highly emetogenic chemotherapy in adults. Cochrane Database Syst Rev 20: CD006272, 2010. 
30. Ezzo JM, Richardson MA, Vickers A, Allen C, Dibble SL, Issell BF, Lao L, Pearl M, Ramirez G, Roscoe J, et al: Acupuncture-point stimulation for chemotherapy-induced nausea or vomiting. Cochrane Database Syst Rev 19: CD002285, 2006.

31. Nakajima S and Katayama T: Running-based pica in rats. Evidence for the gastrointestinal discomfort hypothesis of running-based taste aversion. Appetite 83: 178-184, 2014.

32. Saito R and Takano Y: Easy method for emesis using rats. Nihon Yakurigaku Zasshi 127: 461-466, 2006 (In Japanese).

33. Scarantino CW, Ornitz RD, Hoffman LG and Anderson RF Jr: On the mechanism of radiation-induced emesis: The role of serotonin. Int J Radiat Oncol Biol Physics 30: 825-830, 1994.

34. Andrews PL and Sanger GJ: Abdominal vagal afferent neurones: An important target for the treatment of gastrointestinal dysfunction. Curr Opin Pharmacol 2: 650-656, 2002.

35. Yuan SY, Vilimas P, Zagorodnyuk VP and Gibbins IL: Novel spinal pathways identified by neuronal c-Fos expression after urethrogenital reflex activation in female guinea pigs. Neuroscience 288: 37-50, 2015.

36. Holland RA, Leonard JJ, Kensey NA, Hannikainen PA and De Jonghe BC: Cisplatin induces neuronal activation and increases central AMPA and NMDA receptor subunit gene expression in mice. Physiol Behav 136: 79-85, 2014
37. Rios M: BDNF and the central control of feeding: Accidental bystander or essential player? Trends Neurosci 36: 83-90, 2013.

38. Unger TJ, Calderon GA, Bradley LC, Sena-Esteves $M$ and Rios M: Selective deletion of Bdnf in the ventromedial and dorsomedial hypothalamus of adult mice results in hyperphagic behavior and obesity. J Neurosci 27: 14265-14274, 2007.

39. Moran TH, Norgren R, Crosby RJ and McHugh PR: Central and peripheral vagal transport of cholecystokinin binding sites occurs in afferent fibers. Brain Res 526: 95-102, 1990.

40. Takeda H, Sadakane C, Hattori T, Katsurada T, Ohkawara T, Nagai $\mathrm{K}$ and Asaka M: Rikkunshito, an herbal medicine, suppresses cisplatin-induced anorexia in rats via 5-HT2 receptor antagonism. Gastroenterology 134: 2004-2013, 2008.

This work is licensed under a Creative Commons

Attribution-NonCommercial-NoDerivatives 4.0 International (CC BY-NC-ND 4.0) License. 